\title{
HL7 v2+: The Future of HL7 Version 2?
}

\author{
Frank Oemig ${ }^{1,2}$, Bernd Blobel ${ }^{2,3,4}$, \\ ${ }^{1}$ Deutsche Telekom Healthcare and Security Solutions GmbH, Mülheim, Germany \\ ${ }^{2}$ HL7 Germany, Cologne, Germany \\ ${ }^{3}$ Medical Faculty, University of Regensburg, Germany \\ ${ }^{4}$ eHealth Competence Center Bavaria, Deggendorf Institute of Technology, Germany
}

\begin{abstract}
Background: HL7 version 2.x is the most popular and most propagated data exchange standard in the world. It is mature and adopted by several IHE Technical Frameworks. Nevertheless it has some weaknesses especially in the way it is documented. Several conformance constructs (optionality/usage and repetitions) are still under discussion although the meaning is unambiguous and clear. The deadline for HL7 v2.9 proposals is over and the next ballot is in preparation for May 2016.

Objectives: Therefore the question arises what will come next? HL7 v2.10? HL7 International is in favor of distributing new releases every year, so that this is a good opportunity to update the representation and documentation of HL7 v2.x while maintaining backward compatibility for running interfaces.
\end{abstract}

\section{Correspondence to:}

Frank Oemig, PhD

Deutsche Telekom Healthcare and Security Solutions GmbH

Address: Mülheim, Germany

E-mail: frank.oemig@t-systems.com
Methods: A semantic analysis of the conformance constructs being used by $\mathrm{HL} 7 \mathrm{v} 2 . x$ allows for developing transition matrices so that the new representation can be automatically generated in large parts. Of course, a new separation into domains require manual support.

Results: This paper demonstrates the new representation form rendered out of the HL7 Comprehensive Database.

Conclusions: Harmonizing HL7 v2.x with other standards in the way it is represented simplifies implementation and therefore supports interoperability among applications.

\section{Keywords}

Communication standards; HL7 version 2; interoperability, versioning; compatibility

\section{EJBI 2016; 12(1):en45-en48}

received: April 19, 2016

accepted: April 29, 2016

published: May 20, 2016

\section{Introduction}

HL7 version 2.x is in use since its inception at the end of the 1980s. It has been adopted by global interoperability initiatives like IHE [1] and is therefore part of several Technical Frameworks. The most prominent versions used by IHE are v2.3.1 and v2.5. Obama's Meaningful Use initiative executed by ONC is based on v2.5.1 with enhancements (pre adoption) stemming from v2.8.1 and v2.8.2. Other countries, e.g., UK, are facilitating v2.4. Worldwide, each vendor is implementing its own mixture of versions.

The community is currently working on HL7 v2.9 combined with a discussion about what will come after v2.9. A lot of efforts have been placed on the maintenance and further development of the different versions for HL7 v2.x during the past two decades leaving a large burden on the individual editors who tried to keep the different MS Word documents in sync. As we know today, we have not been successful in that regard all the time, an improvement appears to be necessary.

Observing the way other standards (e.g. HL7 V3®)and FHIR@ 2]) are written, the time has come to take the opportunity for a major step forward and getting rid of old-fashioned conformance constructs. The first step will be done with HL7 v2.9 where chapter 2C (Vocabulary) will not be maintained by hand any more, but generated from a database (Figure 1]. An HL7 project 3] (running for four years now) has examined all tables across all v2.x versions and provided detailed feedback about the consistency, asked for clarification and suggested necessary technical corrections. The ultimate target of this project is a common vocabulary model and future maintenance across all HL7 standards in an equal way. The result of this project is worth another paper and would lead to far here. For Hl7 v2+ only the major achievements in consolidating the tables into a solid vocabulary model consisting 
of vocabulary domains, value sets and codesystems as input into HL7 v2+ are taken.

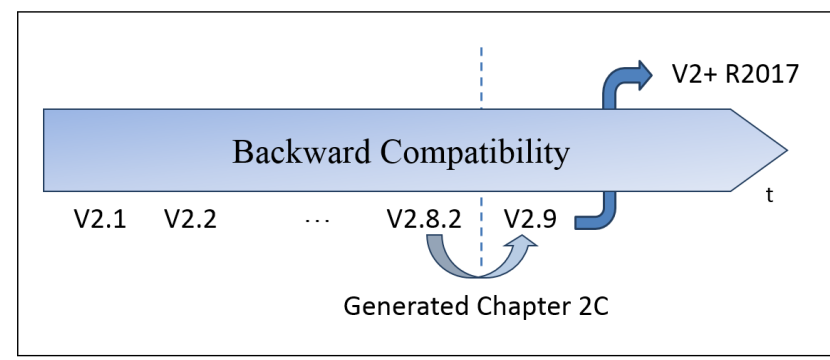

Figure 1: Transition to HL7 v2+.

The next step is the complete generation of the standard from a database. The best input is the HL7 Comprehensive Database that contains the whole documentation already [4].

\section{Methods}

A thorough analysis 5 of the conformance constructs used by HL7 v2.x supports the conclusion that a migration to a better and common representation is possible. A first step is to isolate implementation aspects from runtime information. As such, the "R" (required) and "RE" (required but may be empty) indicators as the cause for long-lasting discussions are to be replaced by "Must Support". Table 1 demonstrates how this conversion is done.

The second conversion concerns the transition of "repetitions" into cardinality. Table 2 demonstrates how.

Using this machinery, a transition can be done automatically. Another algorithm can be used to convert the Abstract Message Syntax into a hierarchical folder structure (Figure 3).

\section{Results}

Following, the current status is presented. Details can be found at 6 . The most prominent visualization of the current enhancements may be seen by the HTML rendering using the newest style that is borrowed from FHIR $\mathrm{R}$. (Figure 2).

The topmost navigation bar allows for accessing the different areas of the specification. Each starting page is new and allows an easy entry into the requested topic. These pages must be created manually because a semantically correct association to a new structure cannot be computed.

\subsection{Overall Representation}

Another improvement is the overall representation form that does not use the Abstract Message Syntax (AMS) any more. As can be seen by Figure 3 instead of different kind of parentheses a hierarchy is introduced that can directly be generated from the "old" specification (AMS) using a 4 phase algorithm. In addition, use of "Must Support" and "Cardinality" flags unifies the appearance.

\begin{tabular}{|c|c|c|c|}
\hline \multirow{2}{*}{$\begin{array}{l}\text { Segment } \\
\text { ORU^R01^ORU R01 }\end{array}$} & \multicolumn{3}{|c|}{ Cardinality Must Support Status } \\
\hline & & & \\
\hline MSH & $1 . .1$ & Yes & \\
\hline \multicolumn{4}{|l|}{ - SFT } \\
\hline - UAC & $0 . .1$ & & \\
\hline PATIENT_RESULT & 1... ${ }^{\star}$ & Yes & \\
\hline PATIENT & $0 . .1$ & & \\
\hline - PID & $1 . .1$ & Yes & \\
\hline PD1 & $0 . .1$ & & \\
\hline PRT & & & \\
\hline NTE & & & \\
\hline NK1 & & & \\
\hline ARV & & & \\
\hline PATIENT_OBSERVATION & & & \\
\hline OBX & $1 . .1$ & Yes & \\
\hline L PRT & & & \\
\hline LISIT & $0 . .1$ & & \\
\hline PV1 & $1 . .1$ & Yes & \\
\hline
\end{tabular}

Figure 3: Message Structure Representation.

The segments are directly hyperlinked to a complete segment documentation which will be provided on a separate page. The segment groups are indicated with a folder icon and an associated segment group name so that all related segments will become a sub-element thereof.

The "Cardinality" and "Must Support" column only indicate a value, if a constraint is placed onto this segment or group. This way the provided information to read the specification is reduced.

\subsection{Segment Representation}

The segments are provided in an enhanced way as well. Again, the usage/optionality information in combination with the repetition indication is replaced by "Must Support" and "Cardinality" and therefore unifies with other standards.

\subsection{Vocabulary Model}

As mentioned in the introduction, a major step forward that is partially provided with v2.9 is the migration to a common vocabulary model. This is accompanied by an enhanced set of meta-data including a movement away from a simple numbering of tables. The common four digit notation will go away in subsequent steps.

This step is visualized by a vocabulary domain name (short name) used in the vocabulary column of the data types and segment definitions (Figure 4). Figure 5 demonstrates the metadata for vocabulary that is still ready for improvements. 
Table 1: Value Set for "Must Support".

\begin{tabular}{|l|l|l|l|}
\hline Value & Description & V2.x compliance & Specialization in profiles \\
\hline $\mathrm{Y}$ & $\begin{array}{l}\text { Must support this element, i.e. a development must } \\
\text { handle this element }\end{array}$ & " $\mathrm{R}$, " $\mathrm{RE}$ " & $\mathrm{Y}$ \\
\hline $\mathrm{N}$ & Element is forbidden & "W", "B", " $\mathrm{X}$ " & $\mathrm{N}$ \\
\hline (empty) & Optional & "O" & $\mathrm{O}, \mathrm{Y}, \mathrm{N}$ \\
\hline
\end{tabular}

Table 2: Value Set for "Cardinality".

\begin{tabular}{|l|l|l|l|}
\hline Value & Description & V2.x Compliance & Rep./\# \\
\hline $0 . .0$ & Forbidden & "W", " $\mathrm{B}$ " & \\
\hline $0 . .1$ & Optional & "RE", "O" & \\
\hline $0 . . \mathrm{n}$ & Optional, repeating n-times & "RE", "O" & "Y"/n \\
\hline $0 . . *$ & Optional, repeating & "O" & "Y" \\
\hline $1 . .1$ & Required & " $\mathrm{R}$ " & \\
\hline $1 . . \mathrm{n}$ & Required, repeating n-times & " $\mathrm{R}^{\prime}$ & "Y" $/ \mathrm{n}$ \\
\hline $1 . . *$ & Required, repeating & " $\mathrm{R}^{*}$ & "Y" \\
\hline $\mathrm{n} . . \mathrm{m}$ & Does not occur yet & & \\
\hline
\end{tabular}

\subsection{Next Steps}

The whole transition process requires a lot of effort until its establishment and acceptance. This paper presents the current status in this long-lasting process realizing that some more steps are necessary:

- Rearranging the presentation into different domains (ADT, billing, orders\&observations, pharmacy, etc.),
- Integration of the two possible encodings (ER7, $\mathrm{v} 2 . \mathrm{xml})$,

- Harmonizing the data types for vocabulary,

- Separating data types, vocabulary and segments into different pages,

- Removing duplicate message definitions by introducing interaction diagrams,

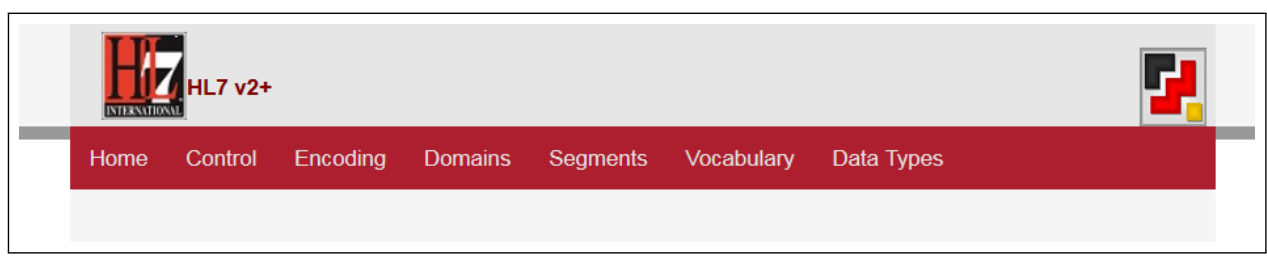

Figure 2: Modern Representation of HL7 v2+.

\begin{tabular}{|c|c|c|c|c|c|c|c|}
\hline Seq\# & $\begin{array}{l}\text { Data } \\
\text { Element }\end{array}$ & Description & Must Support & Cardinality & Length & C.LEN Vocabulary & $\begin{array}{l}\text { Data } \\
\text { Type }\end{array}$ \\
\hline 1 & 00104 & Set ID - PID & & 0.1 & $1 . .4$ & & SI \\
\hline 2 & 00105 & Patient ID & $\begin{array}{l}\text { No } \\
\text { (withdrawn) }\end{array}$ & & & & \\
\hline 3 & 00106 & Patient Identifier List & Yes & $1 . .1$ & & & $c x$ \\
\hline 4 & 00107 & Alternate Patient ID - PID & $\begin{array}{l}\text { No } \\
\text { (withdrawn) }\end{array}$ & & & & \\
\hline 5 & 00108 & Patient Name & Yes & $1 . .1$ & & nameType2 & XPN \\
\hline 6 & 00109 & Mother's Maiden Name & & $0 . .1$ & & & XPN \\
\hline 7 & 00110 & Date/Time of Birth & & 0.1 & & & DTM \\
\hline 8 & 00111 & Administrative Sex & & 0.1 & & administrativeSex & CWE \\
\hline 9 & 00112 & Patient Alias & $\begin{array}{l}\text { No } \\
\text { (withdrawn) }\end{array}$ & & & & \\
\hline 10 & 00113 & Race & & $0 . .1$ & & race & CWE \\
\hline 11 & 00114 & Patient Address & & $0 . .1$ & & & XAD \\
\hline 12 & 00115 & County Code & $\begin{array}{l}\text { No } \\
\text { (withdrawn) }\end{array}$ & & & & \\
\hline 13 & 00116 & Phone Number - Home & No (backward) & & & & XTN \\
\hline
\end{tabular}

Figure 4: PID segment with new Representation. 
- Including other artifacts like XML schemas for conformance profiles, and

- Perhaps allowing for a dynamic behavior, e.g., when working with message structures.

\section{Discussion}

Advancing standards for error correction, scope extension, adoption of new use case, introduction of new components or parameters, etc., results in versioning specifications which contradicts to harmonization and therefore interoperability. Additionally, the presentation style has been changing, and finally each version might be implemented differently, leading to chaotic conditions. To overcome the problems, among others a) a solid vocabulary model representing vocabulary domains, value sets and codesystems must be established, b) the variety of semantics for the value sets must be reduced and simplified, c) the concept representation for the different specifications must be formalized towards a specific ICT ontology, d) automation of specification development process to avoid individual interpretations and inconsistencies is inevitable.

Although this change in representing and providing the standard does not impact any running interface, some kind of retention and therefore rejection is anticipated. Of course, a change in writing the standard is always accompanied with further education requiring time.

\section{Conclusions}

The discussion performed in the paper deals with the improvement of standard specification and implementation processes for enabling interoperability, focused on the ICT domain. The authors presented ongoing developments for HL7 v2+ towards specification harmoniza- tion which will pay off in the near future leading to better implementations and more interoperability. When not limiting interoperability to that domain, but integrating also the supported, ICT independent business domains and their stakeholders, alternatives have been developed, which are even more generic than the presented approach and cover this as well. For more information, see, e.g., [7.

\section{Acknowledgement}

The authors are indebted to thank their colleagues from HL7 International for their support and collegial cooperation.

\section{References}

[1] Integrating the Healthcare Enterprise. IHE Technical Frameworks: www.ihe.net, last accessed January 2016.

[2] Health Level 7 International, Inc. HL7 FHIR: Fast Healthcare Interoperability Resources, www.hl7.org/fhir, lastaccessedJanuary2016

[3] Health Level 7 International, Inc. HL7 V2 Codetable Project: V2 code table versioning and alignment to V3 vocabulary model, http://www.hl7.org/special/Committees/projman/ searchableProjectIndex.cfm?action=edit\&ProjectNumber= 874

[4] Health Level 7 International, Inc. HL7 Comprehensive Database. www.hl7.org

[5] Oemig F, Snelick R: The Conformance Book: The Foundation of Healthcare Interoperability: Conformance and Testing of Data Exchange Standards, Springer International Publishing $\mathrm{AG}$, in print.

[6] Health Level 7 International, Inc. HL7 Refactored: www.hl7. eu/refactored/HL7.html, last accessed January 2016.

[7] Blobel B., Oemig F. A Reference Architecture for Standards Interoperability. European Journal of Biomedicine and Healthcare 2016; 12(1):en3-en6

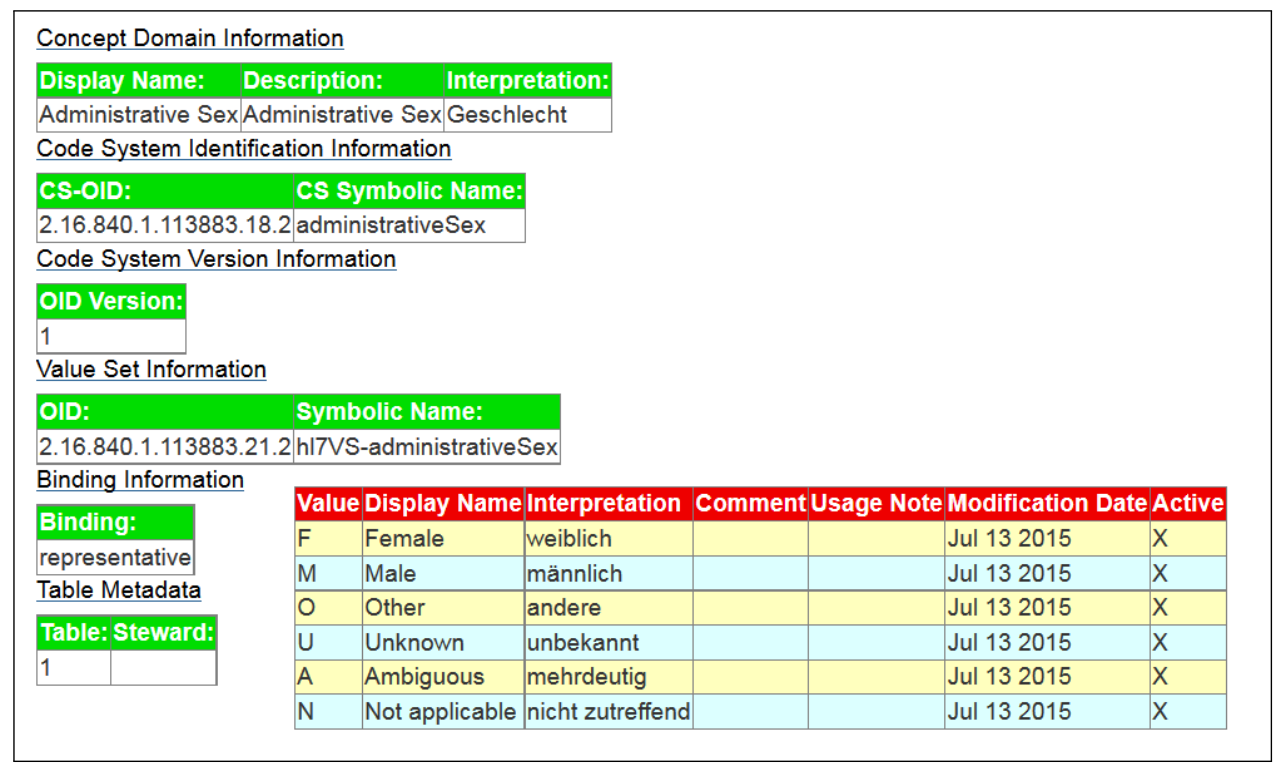

Figure 5: Meta-Data for Vocabulary. 